\title{
Continuous wavelet transform
}

In this section we describe step-by-step our empirical wavelet mode decomposition. First, since the raw signals consist in unevenly spaced time series, each dataset is re-sampled (using linear interpolations when necessary) at the frequency of one observation every ten years and centered so that it has zero mean.

The Lomb-Scargle periodograms plotted in Fig.5 (see text) are obtained with the plomb Matlab function from the raw (irregularly spaced) signals.

The rest of this work is based on the continuous wavelet transform (CWT); self-made codes implemented for Scilab are available upon request (see also the toolbox ...). The main waveletrelated principles involved in this study are inspired from (Deliège et al., 2016; Nicolay S., 2011; Nicolay et al., 2009)] and are summarized below. More information about wavelets and the CWT can be found in e.g. (Daubechies I., 1992; Mallat S., 1999; Meyer Y., 1993; Torrence and Compo, 199).

Given a wavelet $\psi$ and a function $f$, the wavelet transform of $f$ at time $t$ and at scale $a>0$ is defined as

$$
W_{f}(t, a)=\int f(x) \bar{\psi}\left(\frac{x-t}{a}\right) \frac{d x}{a}
$$

where $\psi^{-}$is the complex conjugate of $\psi$. In the context of time-frequency analysis, it is recommended to use a wavelet well-located in the frequency domain ([7]). In this work, we use the wavelet $\psi$ defined by its Fourier transform as

$$
\hat{\psi}(\nu)=\sin \left(\frac{\pi \nu}{2 \Omega}\right) \mathrm{e}^{\frac{-(\nu-\Omega)^{2}}{2}}
$$

with $\Omega=\pi \sqrt{ } 2 / \mathrm{n} 2$, which is similar to the Morlet wavelet but with exactly one vanishing moment ([5]). Since $|\hat{\psi}(\nu)|<10^{-5}$ if $\nu \leq 0, \psi$ can be considered as a progressive wavelet (i.e. is zero for negative arguments) and thus allows an easy recovery of trigonometric functions. Indeed, if $f(x)=$ $\cos (\omega x)$, then

$$
W_{f}(t, a)=\frac{1}{2} \mathrm{e}^{i t \omega} \overline{\hat{\psi}(a w)} .
$$

Therefore, for a fixed time $t$, if $a^{*}$ stands for the scale at which the function

$a \rightarrow\left|W_{f}(t, a)\right|$ reaches its maximum, then we have $a^{*} \omega=\Omega$. Consequently, the value of $\omega$ can be obtained (if unknown) and $f$ is recovered with the real part of its CWT: $f(x)=2 \Re\left(W_{f}\left(x, a^{*}\right)\right)$.

Drawing on this principle, the general scheme for extracting the dominant components of a given function $f$ is the following.

1. The CWT of $f$ is computed and the application $(t, a) \rightarrow\left|W_{f}(t, a)\right|$ can be seen as a timefrequency representation of $f$. 
2. The wavelet-based spectral composition of $f$, also called wavelet spectrum of $f$, i.e. the wavelet counterpart of its Fourier spectrum, is obtained as

$$
\Lambda: a \rightarrow E_{t}\left|W_{f}(t, a)\right|
$$

where $E_{t}$ denotes the mean over time.

3. For each scale $s$ at which $\Lambda$ reaches a local maximum, a component $c_{s}$ is extracted from $f$ as $c_{s}(x)=2 \Re\left(W_{f}(x, s)\right)$.

By adding the modes obtained this way, the CWT allows an almost complete reconstruction of $f$ with smooth amplitude modulated-frequency modulated (AM-FM) components. However, it is slightly different in practice because the signal considered has a finite length and thus has to be padded at its edges. The chosen padding (zero-padding in the present case) irremediably flaws a certain proportion of the wavelet coefficients, which creates a so-called cone of influence (see e.g. Deliège and Nicolay, 2016). Even though it is generally impossible to correct exactly the border effects, noticeable improvements can be made to sharpen the edges of the components extracted and the reconstruction of the initial signal by iterating the process described above. More precisely, the technique used in this work in complement to the previous algorithm is the following.

Let us imagine that the components of interest are located at scales $(s i) i \in I$ (for some set of indices I). Then, at the first iteration of steps 1 to 3 we extract $\left(c^{1}{ }_{i}\right) i \in I$ as $c_{i}{ }^{1}(x)=2 \Re\left(W_{f}(x, s i)\right)$. We now perform the CWT and the extraction at the same scales $\left(s_{i}\right)_{i} \in I$ but with

$$
f_{1}=f-\sum_{i \in I} c_{i}^{1}
$$

and get the modes $\left(c^{2}\right)_{i \in I}$, then again repeat the process with $f_{2}=f_{1}-\sum_{i \in I} c^{2}{ }_{i}$, and so on. The iterations stop when the components extracted are not significant anymore, i.e. at iteration $J$ if

$$
\max \left\{\left\|c_{i}^{J}\right\|, i \in I\right\}<\alpha \max \left\{\left\|c_{i}^{1}\right\|, i \in I\right\}
$$

where $\|$.$\| denotes the energy (square of L^{2}$ norm) of a signal and $\alpha$ is a threshold chosen as 0.01 .

The final components $\left(c_{i}\right)_{i} \in I$ are then obtained as

$$
C i=\sum_{j=1}^{J} c_{i} .
$$

Although most of the energy of $f$ has already been drained at the end of the procedure described in this section, it may turn out useful to examine what kind of information still remains in $f$. For that purpose, the whole method can be applied to $f-\sum_{i \in l} c_{i}$ to find out which frequencies might have been hidden behind the dominant ones and thus obtain "secondary components", if any. 


\section{References}

Daubechies I.: Ten Lectures on Wavelets. SIAM, 1992

Deliège, A. and Nicolay, S.: A New Wavelet-Based Mode Decomposition for Oscillating Signals and Comparison with the Empirical Mode Decomposition, 13th International Conference on Information Technology, 959-968, 2016.

Mallat, S.: A Wavelet Tour of Signal Processing. Academic Press, 1999.

Meyer, Y.: Wavelets and Operators, volume 1. Cambridge University Press, Cambridge, 1993. Nicolay, S.: A wavelet-based mode decomposition. European Physical Journal B, 80, 223-232, 2011.

Nicolay, S., Mabille, G., Fettweis, X., and Erpicum, M.: 30 and 43 months period cycles found in air temperature time series using the Morlet wavelet method. Climate dynamics, 33(7),11171129, 2009.

Torrence, C. and Compo, G.: A practical guide to wavelet analysis. Bulletin of the American Meteorological Society, 79, 61-78, 1998. 
Original data (PN trace element contents, sunspot number, and temperature) were filtered according to the dominant periods revealed by the CWT (Fig.1-5).

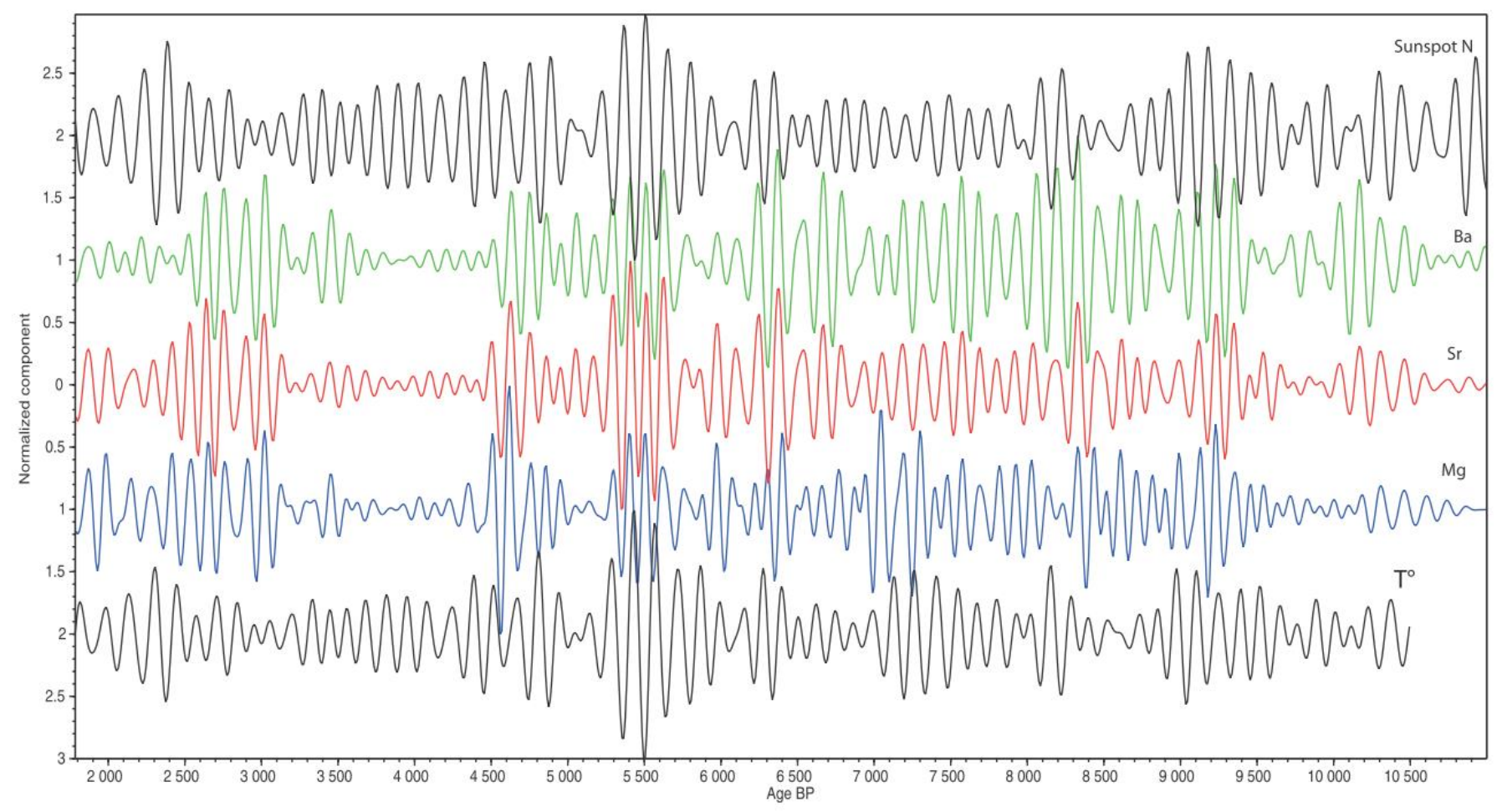

Figure 1: the Gleissberg cycle (70-100 yr) 


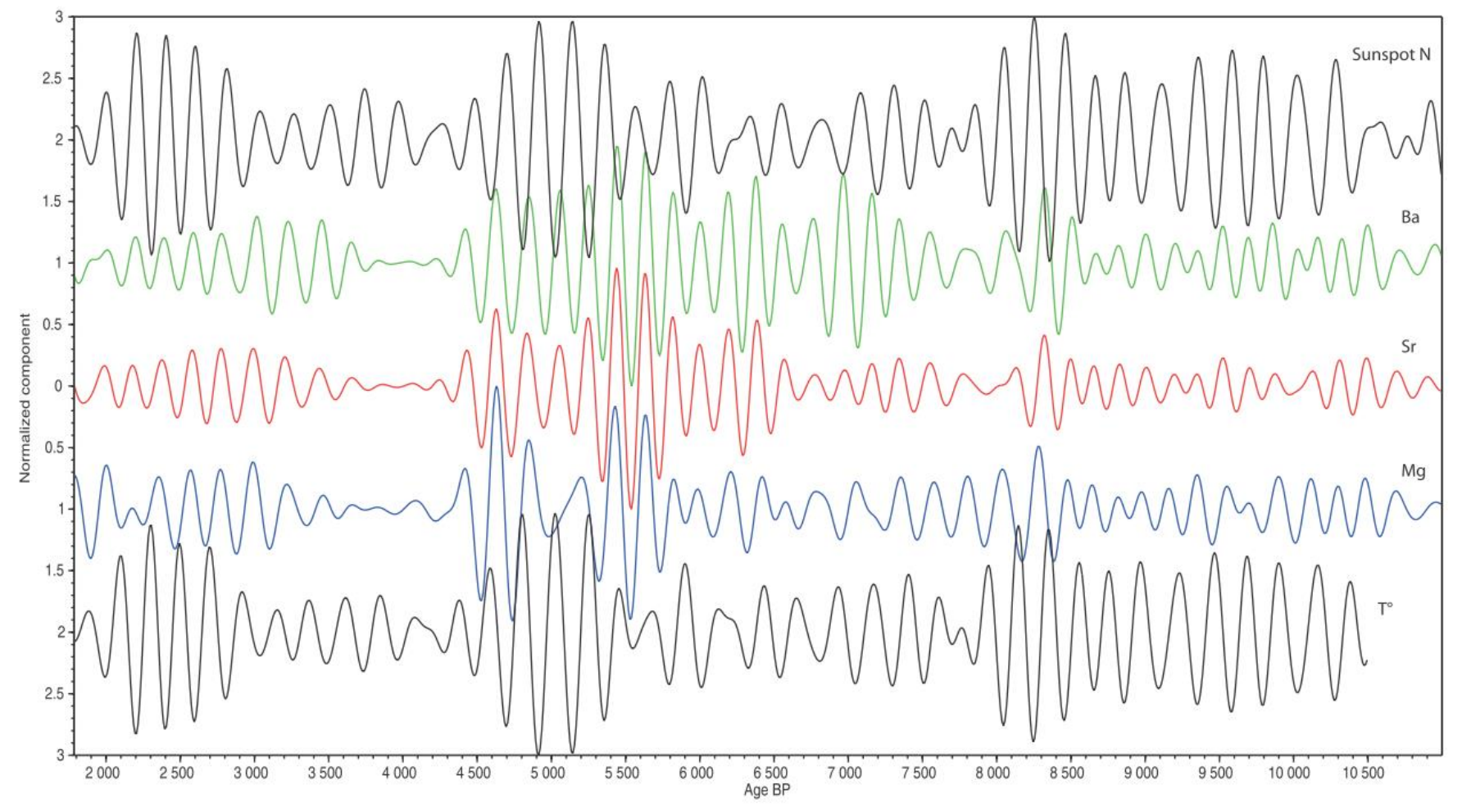


Figure 2: de Vries cycle (200-210 yr)

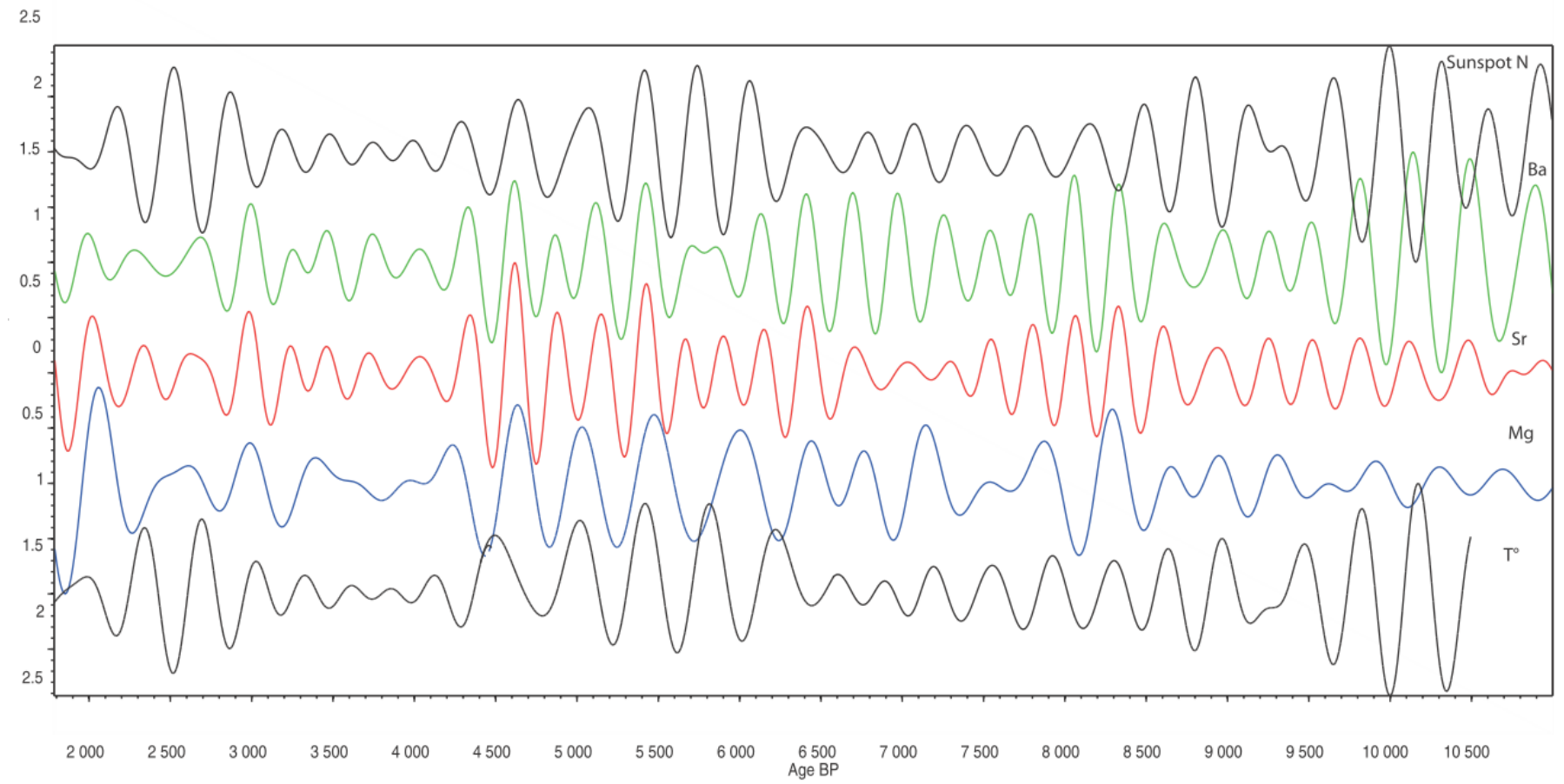

Figure 3: Unnamed cycles $350 \mathrm{yrs}$ 


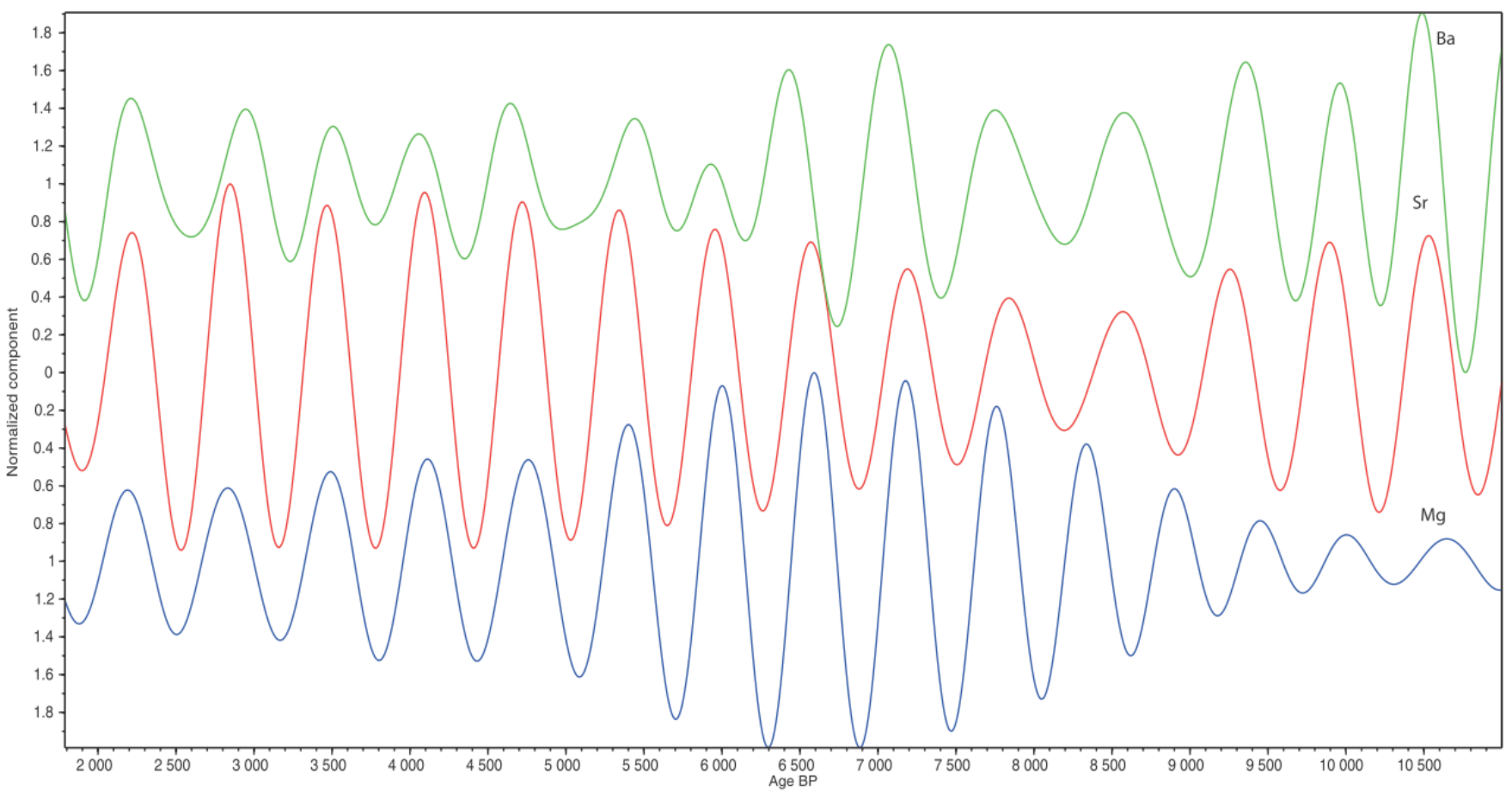

Figure 4: Unnamed cycles 550 yrs

7 


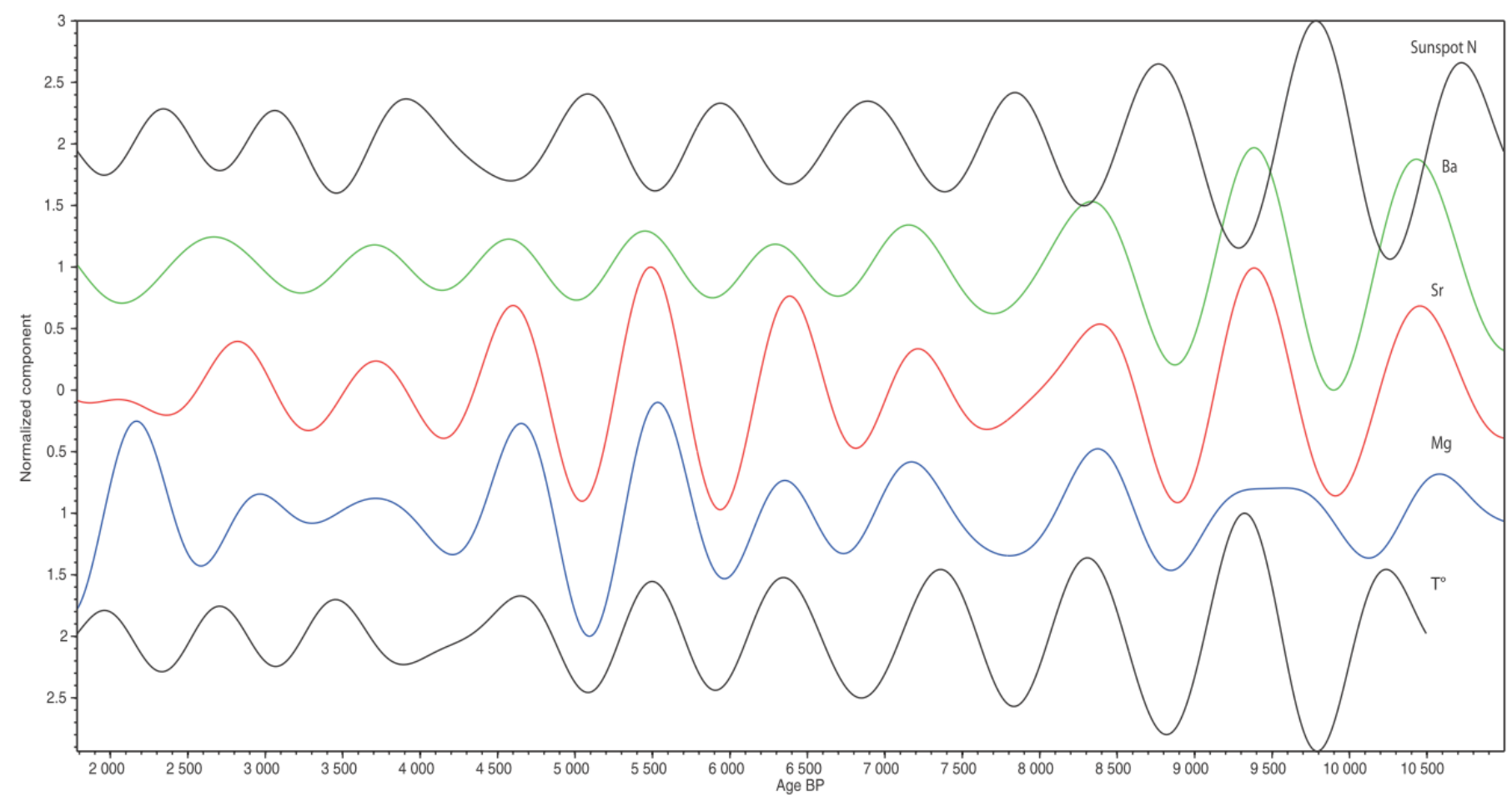

Figure 5: Eddy cycle (1000 yr) 